\title{
CONTRIBUIÇÃO MINERALÓGICA PARA O ESTUDO DE RELEVÂNCIA DE CAVERNAS FERRÍFERAS DA SERRA NORTE, CARAJÁS-PA
}

\author{
A. R. L. ALBUQUeRQUE ${ }^{1 *}$, S. P. A. PAZ ${ }^{1}$, D. F. GONÇALVES ${ }^{2}$, R. S. ANGÉliCA ${ }^{1}$ \\ ${ }^{1}$ Universidade Federal do Pará, Instituto de Geociências, Programa de Pós-Graduação em Geologia e \\ Geoquímica, Laboratório de Caracterização Mineral \\ ${ }^{2}$ Gerência de Meio Ambiente Ferrosos Norte-Vale \\ alanalbuquerquegeo@gmail.com*
}

Submetido16/10/2017 - Aceito 21/11/2017

DOI: $10.15628 /$ holos.2017.6434

\section{RESUMO}

A Província Mineral de Carajás representa uma das maiores províncias minerais do mundo e abriga o maior parque de cavernas em rochas ferríferas do Brasil.Por conta da intensa atividade de extração de minério de ferro em áreas cavernícolas, novas políticas de controle ambiental estão sendo implementadas para contrabalançar a atividade econômica com a preservação do patrimônio ambiental. Dessa maneira, a catalogação e a classificação do grau de relevância de cavernas passaram a ser critérios de licenciamento ambiental para atividades minerarias. Nesse contexto, o presente trabalho teve por objetivo investigar os espeleotemas fosfáticos de cavernas ferríferas de Carajás, com o intuito de detalhar seus constituintes minerais e contribuir com os estudos de suas relevâncias. Cinco cavernas de um platô da Serra de
Carajás foram selecionadas para esse estudo. Os dados petrográficos e mineralógicos permitiram a identificação de quatro minerais fosfáticos: leucofosfita, esfeniscidita, strengita e fosfosiderita. A análise de calorimetria exploratória diferencial (DSC) foi essencial para distinguir dois grupos de fosfatos existentes: leucofosfita-esfeniscidita, com dois eventos endotérmicos, 323,4 e $400^{\circ} \mathrm{C}$, atribuídos à remoção de $\mathrm{OH}$ e $\mathrm{NH}_{4}$, respectivamente, os quais são ausentes no grupo strengita-fosfosiderita. As análises químicas, óticas e de difração de raios $X$ associadas às análises térmicas revelaram uma nova ocorrência de esfeniscidita em ambiente cavernícola, atestando a necessidade da preservação de duas cavernas da Serra Norte, Carajás.

PALAVRAS-CHAVE: Caverna ferrífera, espeleotema fosfático,esfeniscidita, Carajás.

\section{MINERALOGICAL CONTRIBUTION TO RELEVANCE-BASED CLASSIFICATION OF CAVES DEVELOPED IN FERRIFEROUS ROCKS FROM SERRA NORTE, CARAJÁS-PA}

\begin{abstract}
The Carajás Mineral Province is one of the largest mineral provinces in the world, and has the largest caves reserve in iron ore in the Brazil. As a result of iron ore intense extraction in cave areas, new environmental policies have been implemented to attempt to balance economic activity and environmental conservation. Thus, the inventory and relevance-based classification of caves were implement as criteria for environmental licensing of mining ventures. In this context, the objective of this research was study the phosphatic minerals of speleothems from Carajás' iron caves, contributing to the relevance-based studies. Five caves were select for this study, along one of plateaus from
\end{abstract}

Serra Norte, Carajás. The petrographic and mineralogical data allowed us to identify leucophosphite, spheniscidite, strengite and phosphosiderite minerals. The Differential Scanning Calorimetry (DSC) analyses allowed us to identify two phosphatic groups: leucophosphite-espheniscidite, which displayed two endothermic effects at 323,4 and $400^{\circ} \mathrm{C}$ attributed to $\mathrm{OH}$ and $\mathrm{NH}_{4}$ removal, respectively, and strengitephosphosiderite, which displayed no such. The chemical optical, and X-ray diffraction analyses in concert with DSC, revealed a new occurrence of spheniscidite in cave environment, highlighting the need to preserve two caves of the Serra Norte, Carajás. 


\section{INTRODUÇÃO}

As cavernas investigadas nesse trabalho ocorrem ao longo das encostas do Platô N4, que é parte integrante da Unidade Geomorfológica Serra Norte, a qual está inserida no maciço residual da Serra dos Carajás, Pará. As cavernas ferríferas de Carajás estão desenvolvidas na interface da crosta laterítica com o saprólito de rochas do Grupo Grão-Pará (jaspilito e rochas máficas associadas) e ocorrem normalmente no entorno ou na área de explotação de minério de ferro de Carajás (Figura 1).

No princípio, os estudos da formação e dos ecossistemas das cavernas ferríferas brasileiras eram puramente de curiosidade científica. Atualmente, mais precisamente a partir de 2008 (pelo decreto $n^{\circ}$ 6.640), a catalogação e a classificação do grau de relevância desses ecossistemas passaram a ser critérios de licenciamento ambiental para implantação e funcionamento de empreendimentos ligados à atividade mineraria (Auler \& Piló, 2015).

Maurity e Kotschoubey (1995) identificaram espeleotemas constituídos de óxidohidróxidos, sulfatos e fosfatos de Fe e Al em cavernas ferríferas da Serra Norte, mais precisamente no platô N1. Eles identificaram a leucofosfita [ $\left.\mathrm{KFe}_{2}\left(\mathrm{PO}_{4}\right)_{2} \mathrm{OH} .2 \mathrm{H}_{2} \mathrm{O}\right]$, strengita e fosfosiderita (polimorfos de $\mathrm{FePO}_{4} \cdot 2 \mathrm{H}_{2} \mathrm{O}$ ), como os minerais constituintes de espeleotemas mais abundantes, porém não houve uma caracterização detalhada dos referidos minerais, bem como não foi identificado a presença de esfeniscidita $\left[\mathrm{NH}_{4} \mathrm{Fe}_{2}\left(\mathrm{PO}_{4}\right)_{2}(\mathrm{OH}) \cdot 2 \mathrm{H}_{2} \mathrm{O}\right]$.

Nesse contexto, uma investigação acerca dos espeleotemas fosfáticos das cavernas ferríferas de Carajás foi realizada com o objetivo de detalhar os minerais fosfáticos mediante dados químicos, petrográficos, em conjunto com dados de difratometria de raios- $\mathrm{X}$, análises térmicas e microscopia eletrônica. Além disso, essa pesquisa visa contribuir com os estudos de relevância das cavernas ferríferas, bem como colaborar com a preservação e resgate do conhecimento acerca dos espeleotemas fosfáticos, visto que envolve impasses entre atividades minerarias e a conservação patrimonial espeleológica.

\section{MATERIAIS E MÉTODOS}

Foram amostrados 26 pontos distribuídos em 5 cavernas situadas no Platô N4 da Serra Norte (Figura 1). Os pontos amostrados consistiram em 5 amostras de laterita fosfatizada, 9 de crosta laterítica e saprólito de jaspilito, e 12 amostras de espeleotemas.

Os aspectos texturais e mineralógicos foram obtidos mediante análises de 17 lâminas delgadas polidas sob microscópio petrográfico da marca LEICA, modelo DM2700 P. Seguido, foram analisados 10 fragmentos indeformados por Microscopia Eletrônica de Varredura (MEV), combinado à análise química qualitativa pontual por Espectroscopia de Dispersão de Energia (EDS). O equipamento utilizado foi o MEV modelo LEO-1430, sob condições de análises de Elétrons Secundários (ES), corrente de feixe de elétrons $90 \mu \mathrm{A}$, voltagem de aceleração constante $20 \mathrm{kv}$, distância de trabalho $15 \mathrm{~mm}$ e tempo de contagem para aquisição de elementos $30 \mathrm{~s}$. 


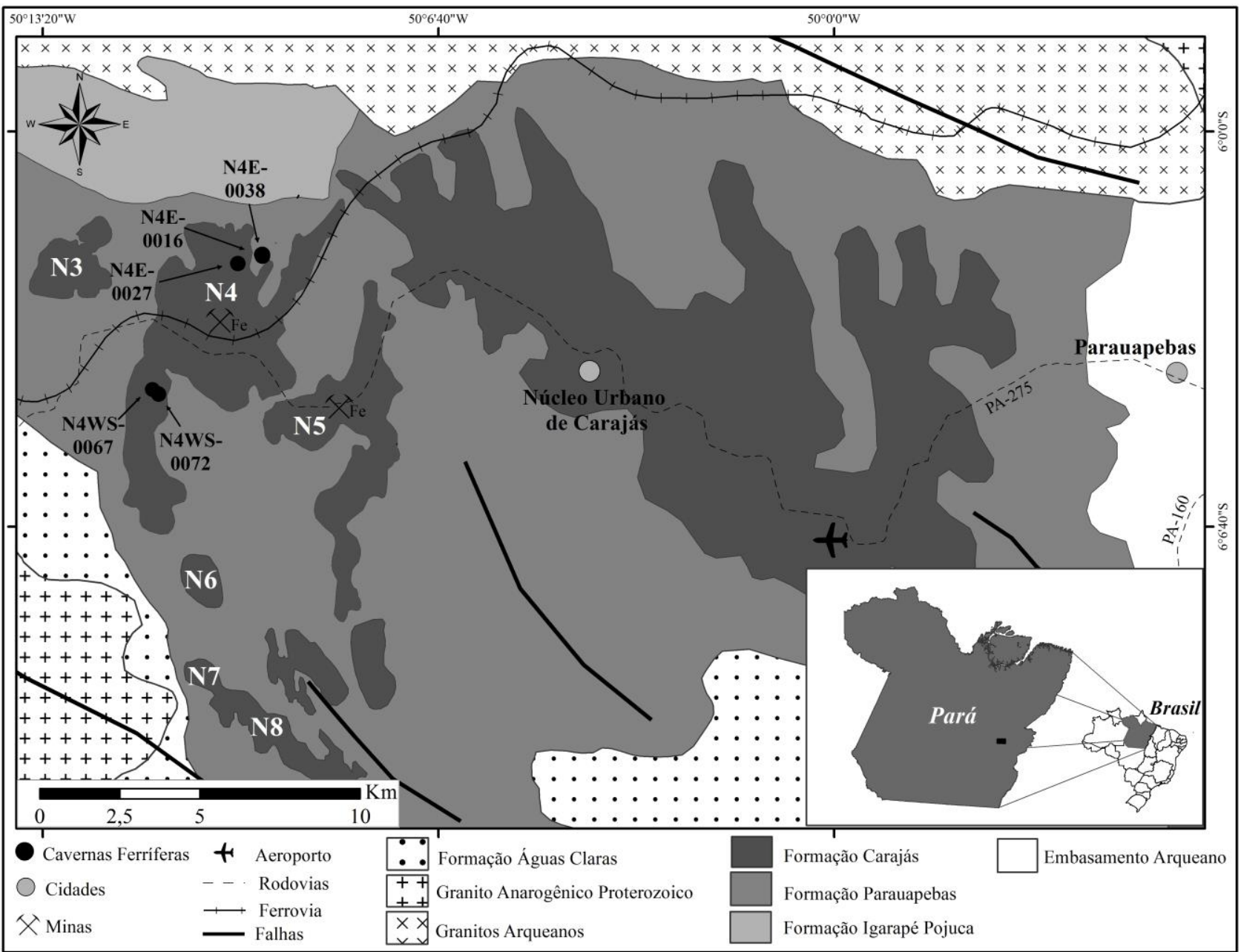

Figura 1: Mapa geológico simplificado com a localização das cavernas estudadas. Fonte: Adaptado de Vasquez e Rosa-Costa (2008).

A identificação mineralógica também consistiu em análises de 30 amostras sob Difratometria de Raios-X (DRX). Foi aplicado o método do pó, utilizando-se difratômetro modelo Empyrean da PANalytical, equipado com anodo de $\mathrm{Co}\left(\mathrm{K} \alpha_{1} 1,790 \AA\right.$ ), filtro $\mathrm{K} \beta$ de $\mathrm{Fe}$, com voltagem de $40 \mathrm{kV}$, corrente de $40 \mathrm{~mA}$, tamanho do passo $0,02^{\circ}$ em $2 \theta$, varredura de 5 o a 750 em $2 \theta$, fenda divergente $1 / 4^{\circ}$ e anti-espalhamento $1 / 2^{\circ}$, máscara $10 \mathrm{~mm}$ e tempo/passo de $70,125 \mathrm{~s}$.

Medidas Calorimétricas Exploratórias Diferenciais (DSC) e termogravimétricas (TG) foram obtidas para 10 amostras de espeleotemas fosfáticos, utilizando um equipamento modelo NETZSCH STA 449F3 Jupiter, com analisador térmico simultâneo da NETStanton Redcroft Ltda, forno cilíndrico vertical de platina, faixa de temperatura entre $25^{\circ} \mathrm{C}$ e $1000^{\circ} \mathrm{C}$, sob fluxo de nitrogênio $50 \mathrm{ml} / \mathrm{min}$, razão de aquecimento de $10^{\circ} \mathrm{C} / \mathrm{min}$ e cadinho de platina como referência.

A caracterização química consistiu na análise de 4 amostras de espeleotemas fosfáticos, na qual os teores dos elementos maiores e menores foram obtidos por Espectrometria de Emissão Óptica com Plasma Acoplado Indutivamente (ICP-OES). A abertura da amostra foi por fusão com metaborato de lítio $\left(\mathrm{LiBO}_{2}\right)$. Os teores de $\mathrm{P}_{2} \mathrm{O}_{5}$ e $\mathrm{NH}_{4}$ foram determinados por Colorimetria usando um espectrofotômetro modelo Cary 50 UV-Vis da Varian. Os valores de Perda ao Fogo (P.F.) foram determinados por calcinação de 2 gramas de cada amostra a $1000^{\circ} \mathrm{C}$ por 1 hora 


\section{RESULTADOS E DISCUSSÃO}

\subsection{Composição química e mineralógica}

As cavernas estudadas no Platô N4 de Serra Norte foram agrupadas em dois grupos: as que ocorrem na porção leste (N4E) e as que ocorrem na porção sudoeste (N4WS).

As cavernas N4E-0016, N4E-0018 e N4E-0027 (Figura 1) desenvolveram-se na interface da crosta laterítica com o saprólito de jaspilito, entre 614 e $660 \mathrm{~m}$ de altitude, e tamanho de 35-139 $\mathrm{m}^{3}$. O saprólito preserva ainda a estrutura reliquiar da rocha matriz e localmente ocorrem porções de jaspilito preservado. A crosta laterítica apresenta aspecto brechoide e porosidade disposta segundo a orientação preferencial das laminações do jaspilito. É composta por clastos angulosos de jaspilito, que são cimentados por óxido-hidróxidos de ferro (Figura 2a). Nessas cavernas ocorrem espeleotemas do tipo crosta e coraloides com dimensões milimétricas e constituídos essencialmente de óxido-hidróxidos de ferro e, em menor proporção, de fosfatos de ferro.

As cavernas N4WS-0067 e N4WS-0072 (Figura 1) desenvolveram-se em crosta laterítica, a 584 e 616 m de altitude, e tamanho de 1689 e $878 \mathrm{~m}^{3}$, respectivamente. A crosta laterítica exibe textura porosa, aspecto brechoide e constitui-se de fragmentos angulosos de jaspilito e nódulos de óxido-hidróxido de ferro (Figura 2a). Localmente, a crosta laterítica encontra-se fosfatizada, onde os fragmentos de jaspilito e nódulos de ferro apresentam bordas substituídas por minerais fosfáticos, ora cristalino, ora amorfo (Figura 2a).

As cavernas do Platô N4WS exibem maior variedade de espeleotemas que as cavernas do Platô N4E, destacando-se: estalactites, estalagmites, crostas e coraloides. Essas feições ocorrem ao longo de paredes e sob a forma de revestimento de piso e teto das cavernas. Nas cavernas do Platô N4WS, os espeleotemas são bem desenvolvidos e constituem-se mineralogicamente de fosfatos de ferro, como: esfeniscidita, leucofosfita, strengita e fosfosiderita (Figura 2a). Essas cavernas abrigam grandes colônias de morcegos, com acumulações centimétricas de guano.

Os fosfatos representam os minerais constituintes de espeleotemas mais abundantes nas cavernas ferríferas de Serra Norte. Os espeleotemas apresentam estruturas porosas, comumente laminadas e concêntricas, com núcleos constituídos normalmente de strengita e fosfosiderita, e bordas compostas por leucofosfita e esfeniscidita. Esses minerais ocorrem como agregados de cristais finos, com coloração cinza esbranquiçado a amarelo pálido (Figura 2b).

Sob análises de microscópia ótica, os minerais fosfáticos não apresentam diferenças texturais, ocorrem como agregados criptocristalinos e, por vezes, com hábitos fibro-radial, prismático, concêntrico, oolítico e esferulítico (Figuras $2 b$ e 2c). Esses minerais são transparentes quando ocorrem de maneira pura, e translúcidos a opacos quando misturados com hidróxidos de ferro (Figuras $2 b$ e $2 c$ ). 


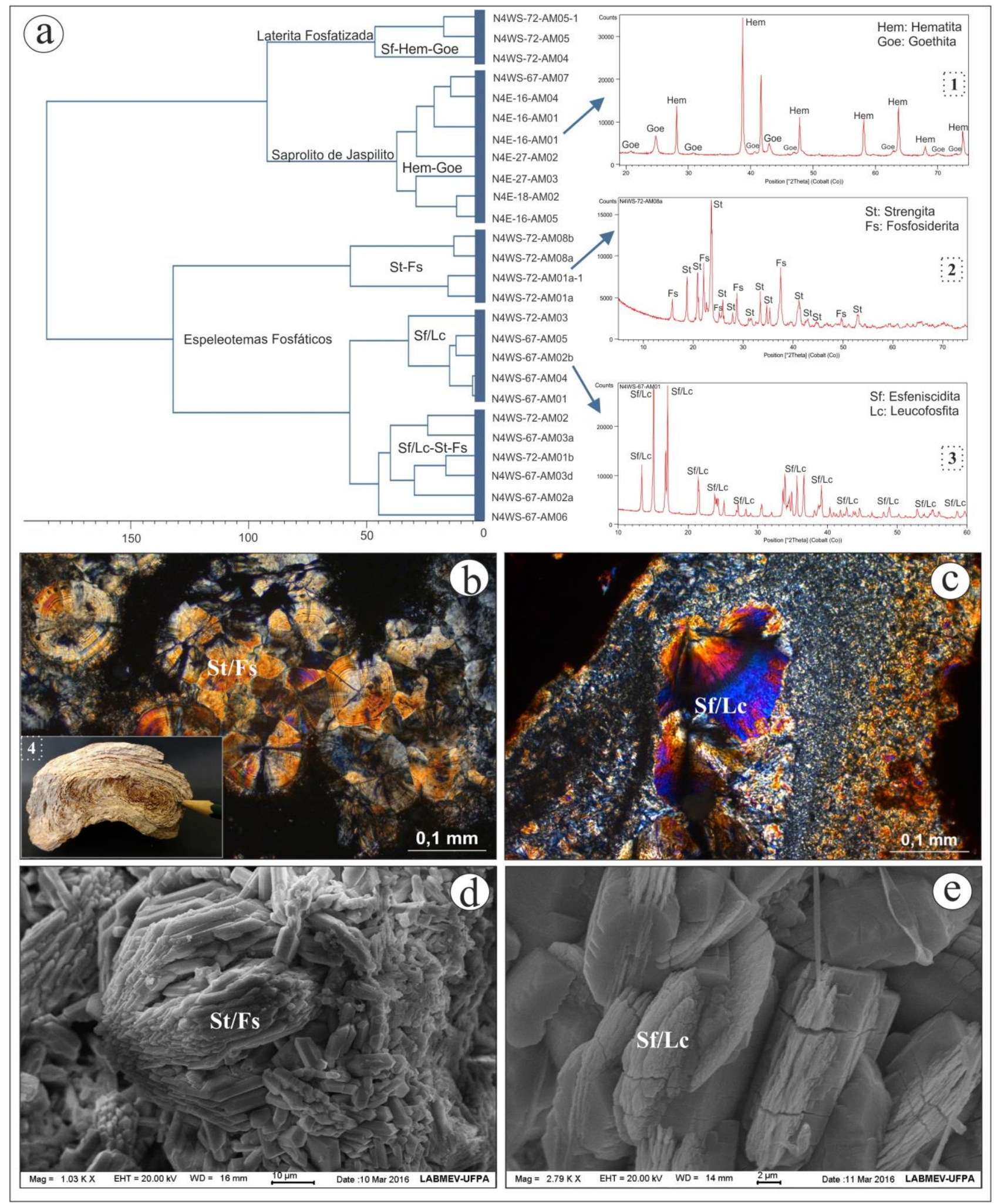

Figura 2: Composição mineralógica dos espeleotemas fosfáticos e das rochas encaixantes das cavernas de Carajás: (a) Análise de cluster com a composição mineralógica da laterita fosfatizada, saprólito de jaspilito/crosta laterítica "1" e dos espeleotemas fosfáticos, ora de forma pura (strengita-fosfosiderita "2" ou esfeniscidita-leucofosfita " 3 "), ora como cristais indiferenciados; (b) Esferólitos indiferenciados de strengita e fosfosiderita (nicóis cruzados) e, em destaque "4", estalactite fosfática; (c) Cristais indiferenciados de leucofosfita-esfeniscidita com estrutura fibroradial (nicóis cruzados); (d) Strengita-fosfosiderita com hábito prismático (ES-MEV); (e) Leucofosfita-esfeniscidita prismática com intercrescimentos de cristais tabulares (ES-MEV). 
A strengita e fosfosiderita podem ser diferenciadas por DRX (Figura 2a), uma vez que são polimorfos de $\mathrm{FePO}_{4} \cdot 2 \mathrm{H}_{2} \mathrm{O}$, ao contrário da leucofosfita e esfeniscidita (Figura 2a) que são isomorfos do sistema cristalino monoclínico, grupo espacial $P 2_{1} / n$ e apresentam solução sólida entre $\mathrm{K}$ e $\mathrm{NH}_{4}$, respectivamente (Wilson \& Bain, 1976). Ao MEV/EDS, não foi possível a diferenciação dos cristais individuais, pois a amônia não é detectada por análises de EDS e a strengita e fosfosiderita são polimorfos e ocorrem associados, assim para os aspectos gerais descritos nesse trabalho, strengita e fosfosiderita, bem como leucofosfita e esfeniscidita foram descritas de maneira conjunta, no qual strengita-fosfosiderita apresentam apenas hábito prismático (Figura 2d), enquanto que leucofosfita-esfeniscidita exibem formas prismáticas com intercrescimento de estruturas tabulares (Figura 2e).

Os espeleotemas fosfáticos são constituídos essencialmente por $\mathrm{Fe}_{2} \mathrm{O}_{3}(39,21-52,94 \%)$, $\mathrm{P}_{2} \mathrm{O}_{5}(38,8-41,28 \%)$ e $\mathrm{H}_{2} \mathrm{O} /$ P.F.(11,95-16,65\%) e, em menor concentração por $\mathrm{NH}_{4}(1,42-3,3 \%)$ e $\mathrm{K}_{2} \mathrm{O}$ $(0,23-0,55 \%)$, veja a Tabela 1 . Esses últimos estando presentes em decorrência à presença de esfeniscidita e leucofosfita, respectivamente. $\mathrm{O}$ aporte de $\mathrm{P}_{2} \mathrm{O}_{5}$, bem como de $\mathrm{NH}_{4}$ e $\mathrm{K}_{2} \mathrm{O}$, para a formação dos espeleotemas fosfáticos, está relacionado à ocorrência de guano nas cavernas ferríferas, uma vez que as rochas hospedeiras das cavernas são empobrecidas nesses elementos (Forti, 2001; Wurster, Munksgaard, Zwart, \& Bird, 2015). Em contrapartida, os elevados teores de $\mathrm{Fe}_{2} \mathrm{O}_{3}$ são herdados das rochas encaixantes das cavernas, as quais são representadas por crosta laterítica e/ou saprólito de jaspilito do Grupo Grão Pará.

Tabela 1: Composição química de quatro amostras de espeleotemas fosfáticos de cavernas ferríferas de Carajás.

\begin{tabular}{c|c|c|c|c|c|c|c|c|c|c|c|c|c}
\hline Amostra & $\mathrm{SiO}_{2}$ & $\mathrm{Al}_{2} \mathrm{O}_{3}$ & $\mathrm{Fe}_{2} \mathbf{O}_{3}$ & $\mathbf{M g O}$ & $\mathbf{M n O}$ & $\mathrm{CaO}$ & $\mathrm{Cr}_{2} \mathrm{O}_{3}$ & $\mathbf{N a}_{2} \mathbf{O}$ & $\mathbf{K}_{2} \mathbf{O}$ & $\mathrm{TiO}_{2}$ & $\mathbf{P}_{2} \mathbf{O}_{5}$ & $\mathbf{N H}_{4}$ & $\mathbf{P . F}_{1000}{ }^{\circ} \mathrm{C}$ \\
\hline 01 & 1,22 & 0,48 & 52,94 & 0,13 & 0,02 & $<0,01$ & $<0,01$ & 0,02 & 0,89 & 0,26 & 39,69 & 2,5 & 11,95 \\
02 & $<0,01$ & 0,18 & 39,89 & 0,17 & 0,03 & $<0,01$ & $<0,01$ & 0,02 & 0,81 & 0,04 & 38,8 & 2,1 & 17,41 \\
03 & $<0,01$ & 0,28 & 40,79 & 0,07 & 0,01 & $<0,01$ & $<0,01$ & 0,02 & 0,55 & 0,06 & 39,12 & 1,42 & 17,92 \\
04 & $<0,01$ & 1,6 & 39,21 & 0,16 & 0,01 & 0,03 & $<0,01$ & 0,02 & 0,23 & 0,02 & 41,28 & 3,3 & 16,65 \\
\hline
\end{tabular}

Nota: Unidade em \% massa; ( < ) valores inferiores ao limite de detecção pelo método de análise utilizado e P.F. perda ao fogo à $1000^{\circ} \mathrm{C}$.

\subsection{Comportamento térmico dos fosfatos de ferro}

\subsubsection{Strengita - Fosfosiderita}

As curvas DSC-TG medidas para amostras de espeleotemas de strengita-fosfosiderita, de temperatura ambiente até $1000^{\circ} \mathrm{C}$, exibiram quatro efeitos térmicos bem definidos (Figura 3a). São eles, de acordo com Arlidge, Farmer, Mitchell e Mitchell (1963), Nathan, Panczer e Gross (1988) e Reale et al. (2003):

(1) Efeito endotérmico fraco e difuso a $\sim 100^{\circ} \mathrm{C}$ está relacionado à saída de água de umidade, com perda de massa 0,9\%;

(2) Efeito endotérmico a $207,9^{\circ} \mathrm{C}$ está associado à remoção de duas moléculas de água estrutural, resultando na perda de massa 19,60\%. A saída das duas moléculas de $\mathrm{H}_{2} \mathrm{O}$ confere caráter anidro a esses minerais, seguido pelo colapso estrutural e formação de fases minerais semi-amorfas; 
(3) Efeito exotérmico a $648,5^{\circ} \mathrm{C}$ é atribuído ao processo de recristalização e formação de nova fase mineral de $\mathrm{FePO}_{4}$ com estrutura tipo high-quartzo;

(4) Efeito endotérmico a $712,8^{\circ} \mathrm{C}$ é conferido pelo processo de decomposição e fusão da amostra, visto que o $\mathrm{FePO}_{4}$ puro é estável até $710^{\circ} \mathrm{C}$.

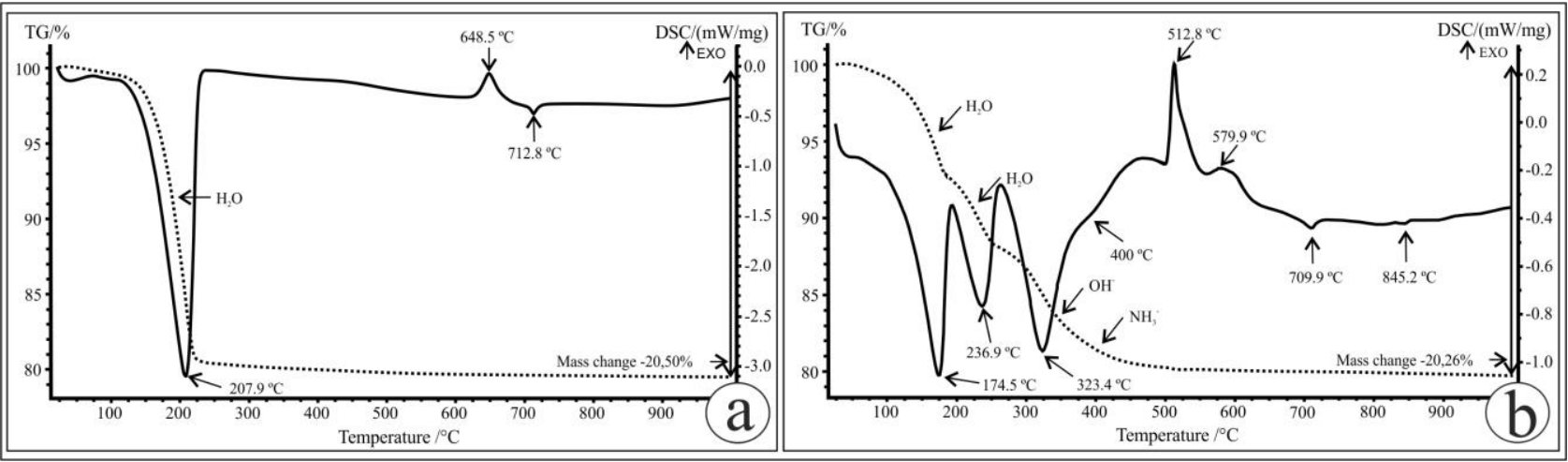

Figura 3: Curvas DSC (linha cheia) e TG (linha pontilhada) reportadas para minerais de strengita-fosfosiderita (a) e para cristais de leucofosfita-esfeniscidita (b).

\subsubsection{Leucofosfita - Esfeniscidita}

As curvas DSC-TG medidas para amostras de leucofosfita-esfeniscidita, de temperatura ambiente até $1000^{\circ} \mathrm{C}$, exibiram efeitos térmicos bem definidos e diferentes da strengitafosfosiderita (Figura 3 b). São eles, de acordo com Arlidge (1963), Wilson e Bain (1976), Marincea, Dumitraş e Gibert (2002), Reale et al. (2003) e Yuan et al. (2008):

(1) Efeito endotérmico fraco e difuso a $100^{\circ} \mathrm{C}$ é atribuído à remoção de água de umidade, com perda de massa 0,32\%;

(2) Efeito endotérmico a $174,5^{\circ} \mathrm{C}$ configura a remoção de uma molécula de $\mathrm{H}_{2} \mathrm{O}$ "livre", geralmente localizada em cavidades estruturais. A remoção dessa molécula representa perda de massa 7,23\%, conferindo-lhe caráter mono-hidratado, porém sem significativo rearranjo estrutural;

(3) Efeito endotérmico a $236,9^{\circ} \mathrm{C}$ está associado à remoção da segunda molécula de $\mathrm{H}_{2} \mathrm{O}$, com perda de massa 4,43\% e formação de mineral anidro;

(4) Efeito endotérmico a $323,4^{\circ} \mathrm{C}$ está relacionado à remoção do grupo $\mathrm{OH}^{-}$, seguido pela saída de $\mathrm{NH}_{3}{ }^{-}$a $400^{\circ} \mathrm{C}$, acarretando uma perda de massa $~ 7,71 \%$. A saída do grupo $\mathrm{OH}^{-}$e da $\mathrm{NH}_{3}{ }^{-}$ resulta no colapso total da estrutura e consequentemente sua completa amorfização. A saída da molécula de $\mathrm{NH}_{3}{ }^{-}$, associada aos outros dados da pesquisa, revelaram a presença de esfeniscidita, a qual foi reportada pela primeira vez por Sauro et al. (2014), seguido por Wurster et al. (2015), porém em caverna quatzítica e em cavernas carbonáticas, respectivamente;

(5) Eventos exotérmicos a $512,8^{\circ} \mathrm{C}$ e $579,9^{\circ} \mathrm{C}$ são atribuídos a recristalização e formação de nova fase mineral de $\mathrm{FePO}_{4}$ com estrutura tipo high-quartzo;

(6) Eventos endotérmicos a $709,9^{\circ} \mathrm{C}$ e $845,2^{\circ} \mathrm{C}$ configuram processos de decomposição e fusão da amostra. 


\section{CONCLUSÕES}

Os minerais fosfáticos são os minerais constituintes de espeleotemas mais abundantes das cavernas ferríferas de Carajás. Os principais minerais ocorrem como strengita, fosfosiderita, leucofosfita e também esfeniscidita. Essa é a terceira ocorrência de esfeniscidita em ambiente cavernícola e a primeira em cavernas ferríferas. A presença de esfeniscidita foi revelada pela concentração de $\mathrm{NH}_{4}$ detectada em análises químicas, e pelo efeito endotérmico a $400^{\circ} \mathrm{C}$, correspondente à saída de $\mathrm{NH}_{3}{ }^{-}$, através de análises térmicas.

Dessa maneira, a presente pesquisa atesta a necessidade de preservação das cavernas N4WS-0067 e N4WS-0072, não só pelo seu rico conteúdo mineralógico e espeleológico, como também por suas grandes dimensões, incomum em rochas de baixa solubilidade, como as rochas ferríferas.

\section{AGRADECIMENTOS}

Agradecimentos ao grupo de pesquisa "Levantamento Geoespeleológico de Cavidades Naturais da Unidade Espeleológica de Carajás, Província Mineral de Carajás-PROCAV", à VALE pela assistência na pesquisa de campo, e ao Programa de Pós-Graduação em Geologia e GeoquímicaPPGG/UFPA e ao Laboratório de Caracterização Mineral-LCM/UFPA por todo suporte laboratorial.

\section{REFERÊNCIAS}

Arlidge, E.Z., Farmer, V.C., Mitchell, B.D., \& Mitchell, W.A. (1963). Infra-red, x-ray and thermal analysis of some aluminium and ferric phosphates. Journal of Applied Chemistry, 13, 17-27.

Auler, A.S., \& Piló, L.B. (2015). Caves and Mining in Brazil: The dilemma of cave preservation within a mining context. In Andreo B et al. (eds). Hydrogeological and Environmental Investigations in Karst Systems. Springer, p. 487-496.

Forti, P. (2002). Biogenic speleothems: an overview. International Journal Speleology,30, 39-56.

Marincea, S., Dumitraş, D., \& Gibert, R. (2002). Tinsleyite in the "dry" Cioclovina Cave (Sureanu Mountains, Romania): the second occurrence. European Journal of Mineralogy, 14, 157-164.

Maurity, C.W., \& Kotschoubey, B. (1995). Evolução Recente da Cobertura de Alteração no Platô NI-Serra dos Carajás-PA. Degradação, pseudocarstificação, espeleotemas. Boletim do Museu Paraense Emílio Goeldi, 7, 331-362.

Nathan, Y., Panczer, G., \& Gross, S. (1988). The thermal analysis of some phosphate minerals: strengite, lipscombite. cyrilovite and goyazite. Thermochimica Acta, 135, 259-266.

Reale, P., Scrosati, B., Delacourt, C., Wurm, C., Morcrette, M., \& Masquelie, C. (2003). Synthesis and thermal behavior of crystalline hydrated Iron (III) phosphates of interest as positive electrodes in Li batteries. Chemistry of Materials, 15, 5051-5058. 
Sauro, F., Waele, J., Onac, B. P., Galli, E., Dublyansky, Y., Baldoni, E., \& Sanna, L. (2014). Hypogenic speleogenesis in quartzite: The case of Corona 'e Sa Craba Cave (SW Sardinia, Italy). Geomorphology, 211, 77-88.

Vasquez, M. L., \& Rosa-Costa, L. T. (2008). Geologia e Recursos Minerais do Estado do Pará: Sistema de Informações Geográficas-SIG: texto explicativo dos mapas Geológico e Tectônico e de Recursos Minerais do Estado do Pará. Escala 1:1.000.000. Relatório Técnico, CPRM, Belém, Brasil.

Wilson, M. J., \& Bain, D. C. (1976). Occurrence of leucophosphite in a soil from Elephant Island, British Antarctic Territory. American Mineralogist, 61, 1027-1028.

Wurster, C. M., Munksgaard, N., Zwart, C., \& Bird, M. (2015). The biogeochemistry of insectivorous cave guano: a case study from insular Southeast Asia. Biogeochemistry, 124, 163-175.

Yuan, A. Q., Wu, J., Huang, Z. Y., Wu, K., Liao, S., \& Tong, Zh. F. (2008). Synthesis of $\mathrm{NH}_{4} \mathrm{FePO}_{4} \cdot \mathrm{H}_{2} \mathrm{O}$ nano-plats via solid-state reaction at low temperature and its thermochemistry properties. Materials Research Bulletin, 43, 1339-1345. 